\title{
Fatty acid profiles and their distribution patterns in microalgae: a comprehensive analysis of more than 2000 strains from the SAG culture collection
}

Imke Lang ${ }^{1,2}$, Ladislav Hodac ${ }^{3}$, Thomas Friedl ${ }^{3}$ and Ivo Feussner ${ }^{1 *}$

\begin{abstract}
Background: Among the various biochemical markers, fatty acids or lipid profiles represent a chemically relatively inert class of compounds that is easy to isolate from biological material. Fatty acid (FA) profiles are considered as chemotaxonomic markers to define groups of various taxonomic ranks in flowering plants, trees and other embryophytes.

Results: The fatty acid profiles of 2076 microalgal strains from the culture collection of algae of Göttingen University (SAG) were determined in the stationary phase. Overall 76 different fatty acids and 10 other lipophilic substances were identified and quantified. The obtained FA profiles were added into a database providing information about fatty acid composition. Using this database we tested whether FA profiles are suitable as chemotaxonomic markers. FA distribution patterns were found to reflect phylogenetic relationships at the level of phyla and classes. In contrast, at lower taxonomic levels, e.g. between closely related species and even among multiple isolates of the same species, FA contents may be rather variable.

Conclusion: FA distribution patterns are suitable chemotaxonomic markers to define taxa of higher rank in algae. However, due to their extensive variation at the species level it is difficult to make predictions about the FA profile in a novel isolate.
\end{abstract}

\section{Background}

The analysis of the overall fatty acid profiles as well as the occurrence of fatty acids (FAs) in different lipid classes in microalgae is an emerging field which is expected to reveal the identification of novel FAs with a variety of new functional groups [1]. Despite a number of reports has been carried out and published, describing the contents as well as the composition of polyunsaturated fatty acids (PUFAs) in mostly marine microalgae [2-4], systematic approaches that include different or even many genera of microalgae and particularly those from freshwaters or terrestrial habitats are still missing [5].

Based on current knowledge, FA composition divides microalgae roughly into two groups, i.e. on one hand the cyanobacteria and green algae (Chlorophyta and Streptophyta) which contain low amounts of FAs, predominantly

\footnotetext{
* Correspondence: ifeussn@gwdg.de

'Georg-August-University, Albrecht-von-Haller-Institute for Plant Sciences,

Department of Plant Biochemistry, Göttingen, Germany

Full list of author information is available at the end of the article
}

saturated and mono unsaturated FAs as well as trace amounts of PUFAs, mostly linoleic acid (LA, 18:2(9Z, $12 Z)$ : where $\mathrm{x}: \mathrm{y}(\mathrm{z})$ is a fatty acid containing $\mathrm{X}$ carbons and $\mathrm{y}$ double bonds in position $\mathrm{z}$ counting from the carboxyl end)). On the other hand Chromalveolate algae contain significant amounts of PUFAs [6].

Among the various biochemical markers, FA or lipid profiles represent a chemically relatively inert class of compounds that is easy to isolate from biological material and FA profiles are considered as chemotaxonomic markers to define groups of various taxonomic ranks in flowering plants, trees and other Embryophytes $[7,8]$.

Beside the identification of novel FAs, some recent studies report on the use of FAs and lipid profiles of algae as biomarkers [1,9-11]. Viso et al. determined profiles of FAs of nine different marine algal groups and they were able to define even species-specific lipid compositions [4]. Moreover they found a roughly taxon specific profile when the cells were cultured under identical growth conditions. Various strains and species of the cyanobacterium Nostoc
C Biomed Central

(c) 2011 Lang et al; licensee BioMed Central Ltd. This is an Open Access article distributed under the terms of the Creative Commons Attribution License (http://creativecommons.org/licenses/by/2.0), which permits unrestricted use, distribution, and reproduction in any medium, provided the original work is properly cited. 
were screened for their FA content and the application of a FA-based cluster analysis has been described for their identification [12].

FA and lipid composition have also been used as biomarkers to distinguish closely related microalgae at the species and the generic levels $[11,13]$. Hitherto no systematic analysis has been carried out on a large scale basis on either the profiles of lipids or FAs in microalgae. Therefore, we determined the FA profiles of all available microalgal strains of the SAG culture collection of microalgae http://www.epsag.uni-goettingen.de which is one of the most diverse and comprehensive resources of microalgae. At present (March 2011) 2291 strains of mainly microscopic algae including a considerable variety of cyanobacteria is available. They comprise almost all phyla and classes of eukaryotic algae, but an emphasis is put on algae from freshwaters and terrestrial habitats.

Distribution patterns of FAs may be valuable also as a proxy to identify certain groups, species and strains of microalgae of particular interest for applied research, i.e. due to the presence of certain FAs and/or high percentages of total FA content. We also tested whether the detected FA distribution patterns are meaningful in a phylogenetic context at various taxonomic levels, i.e. to define taxonomic groups of microalgae by their FA patterns. It would assist predicting FA content and/or presence of other valuable compounds if the phylogenetic relationships of algae were reflected in their FA distribution patterns.

Here the focus was set on esterified long chain FAs (C14 - C-24), which were analysed via Gas chromatography (GC) with or without mass spectrometry (MS). The large number of data obtained, were added into a database to document the FA profiles of the studied microalgal strains.

\section{Results and Discussion}

\section{A database of FA profiles from diverse microalgae}

The characterisation of FA profiles of the SAG microalgal strains was performed by screening long chain FAs (C-14 - C-24) esterified within lipids. A total of 2076 culture strains from the SAG (equal 91\% of the SAG's holding) were screened. A database was established which contained all identified FAs and some other hydrophobic metabolites. An overview of all substances identified in the algal strains screened is shown in Table 1. A total of 86 different substances were identified by mass spectrometry, 76 of which represent methyl esters of FAs. Out of the 76 fatty acids, 36 substances were identified by their mass spectrum and by retention time according to a standard substance, and the other 40 fatty acids were identified by their mass spectra only. The remaining 10 substances were identified by their mass spectra only as well. In comparisons with a standard substance, the compound was identified by comparison to mass spectra with highest similarity to the proposed substance in the MS-library (Nist02 or Wiley98). By this some methyl esters of branched FAs were detected, for example 12-methyl-14:0 or 3, 7, 11, 15-tetramethyl-16:0. Whereas for most of the FAMEs, authentic standards or MS references were available, for some other substances only "best hit" identification was possible. The DMOX derivatives enabled the identification of the remaining 12 FAMEs. Unidentified substances have yet to be verified with authentic standards, which are not available at this time point. The complete database is shown as additional file 1 .

Bacteria in algal cultures (as contaminations or sometimes even through symbiosis) are well known and can be found in culture strains of almost any algal culture collection. Only a small fraction (about 20\%) of the studied SAG strains may be in axenic state. Therefore, also the FA content of the contaminating bacteria may have contributed to the obtained FA profile. To test this, we measured methyl-15:0 and methyl 17:0 that are regarded as markers for bacterial contaminations [4]. Only 34 strains out of the 2076 analyzed strains contained small amounts methyl15:0. This observed low rate of contaminating bacteria was supported by microscopic controls which are routine in the perpetual maintenance of algal strains (data not shown). In summary, we conclude that only $1-2 \%$ of the strains may have been contaminated and that there is only a minor influence of bacterial contaminations on the observed algal culture FA profiles.

In addition we compared the measured major FA profiles of 10 randomly chosen strains from different classes with published data (Table 2), and it should be noted that only one out of the 10 strains that were chosen from the published data originated from the SAG collection. For 6 strains the FA profiles were very similar. In case of the 4 remaining strains major differences were observed in the degree of desaturation of the FAs with different chain lengths, which may be explained by the different cultivation conditions used in the different studies.

\section{Patterns of fatty acid composition}

FAME profiles were rather different among strains. As an example, FAME profiles from four different genera, i.e. Chroococcus (Cyanobacteria), Closteriopsis (Chlorophyta, Trebouxiophyceae), Pseudochantransia (Rhodophyta) and Prymnesium (Chromalveolates, Haptophyta) are presented in Figure 1. Therefore it was anticipated to recover certain different FA distribution patterns between phyla, classes and genera of microalgae. In addition, it was tested whether differences in FA patterns can also be found for groups at lower taxonomic rank, i.e. between species of the same genus or even among multiples isolates of the same species. 
Table 1 Overview of the FAMEs identified and other substances found in the analysed SAG microalgal strains

\begin{tabular}{|c|c|c|}
\hline \multicolumn{3}{|l|}{86 substances, 76 methyl esters of FAs } \\
\hline methyl esters of saturated straight-chain FAs & methyl esters of branched chain FAs & methyl esters of monoenoic FAs \\
\hline$\overline{14: 0}$ & 12-methyl-14:0 & $14: 1(7 Z)$ \\
\hline $16: 0$ & 13-methyl-14:0 & $14: 1(9 Z)$ \\
\hline $17: 0$ & 14-methyl-15:0 & $15: 1(10 Z)$ \\
\hline 18:0 & 14-methyl-16:0 & $16: 1(5 Z)$ \\
\hline 19:0 & methyl-3, 7, 11, 15-tetramethyl-16:0 & $16: 1(7 Z)$ \\
\hline $20: 0$ & 16- o. 15-methyl-17:0 & $16: 1(9 Z)$ \\
\hline $21: 0$ & 17-methyl-18:0 & $16: 1(11 Z)$ \\
\hline $22: 0$ & $6,10,14$ trimethyl-2-pentadecanone & $17: 1(8 Z)$ \\
\hline $23: 0$ & & $17: 1(9 Z)$ \\
\hline \multirow[t]{2}{*}{ 24:0 } & & 17:1 (10Z) \\
\hline & & 18:1 (9E) \\
\hline methyl esters of dienoic FAs & methyl esters of trienoic FAs & $18: 1(9 Z)$ \\
\hline $15: 2$ & $16: 3(4 Z, 7 Z, 10 Z)$ & $18: 1(11 Z)$ \\
\hline $16: 2(7 Z, 10 Z)$ & $16: 3(6 Z, 9 Z, 12 Z)$ & 19:1 (11Z) \\
\hline $16: 2(9 Z, 12 Z)$ & $16: 3(7 Z, 10 Z, 13 Z)$ & $20: 1(11 Z)$ \\
\hline $17: 2(7 Z, 10 Z)$ & $17: 3$ & 22:1 (13Z) \\
\hline $17: 2(9 Z, 12 Z)$ & $18: 3(5 Z, 9 Z, 12 Z)$ & $24: 1(15 Z)$ \\
\hline $18: 2(6 Z, 9 Z)$ & $18: 3(6 Z, 9 Z, 12 Z)$ & \\
\hline $18: 2(8 Z, \times Z)^{*}$ & $18: 3(8 Z, 11 Z, 14 Z)$ & \\
\hline $18: 2(9 E, 12 E)$ & $18: 3(9 Z, 12 Z, 15 Z)$ & \\
\hline $18: 2(9 Z, 12 Z)$ & $19: 3$ & \\
\hline $18: 2(9 Z, 14 Z)$ & $19: 3$ & \\
\hline $18: 2(11 Z, 14 Z)$ & $20: 3(7 Z, 10 Z, 13 Z)$ & \\
\hline $19: 2(9 Z, 12 Z)$ & $20: 3(8 Z, 11 Z, 14 Z)$ & \\
\hline $20: 2(11 Z, 14 Z)$ & $20: 3(11 Z, 14 Z, 17 Z)$ & \\
\hline $22: 2(13 Z, 16 Z)$ & $22: 3$ & \\
\hline methyl esters of tetra-, penta-, and hexaenoic FAs & other substances & \\
\hline $16: 4(4 Z, 7 Z, 10 Z, 13 Z)$ & (8Z, 11Z)-heptadeca-8, 11-dienal & \\
\hline $16: 4(6 Z, 9 Z, 12 Z, 15 Z)$ & 3-(3, 5-ditertbutyl-4-hydroxyphenyl) propionate & \\
\hline $18: 4(5 Z, 9 Z, 12 Z, 15 Z)$ & $3,7,11,15$-tetramethyl-2-hexadecen-1-ol & \\
\hline $18: 4(6 Z, 9 Z, 12 Z, 15 Z)$ & 8-(2-octylcyclopropyl) octadecanoate & \\
\hline $19: 4$ & 2, 3, 4, 5- tetramethyl-3-hexen & \\
\hline $20: 4(5 Z, 8 Z, 11 Z, 14 Z)$ & $(5 Z, 8 Z, 11 Z)-15,16$ epoxy 5, 8, 11-octadecadienoate & \\
\hline $20: 4(8 Z, 11 Z, 14 Z, 17 Z)$ & Tetradecanamide & \\
\hline $22: 4(7 Z, 10 Z, 13 Z, 16 Z)$ & Hexadecanamide & \\
\hline $18: 5(3 Z, 6 Z, 9 Z, 12 Z, 15 Z)$ & (9Z)-Octadecenamide & \\
\hline $20: 5(5 Z, 8 Z, 11 Z, 14 Z, 17 Z)$ & 9, 10-methylene tetradecanoate & \\
\hline \multicolumn{3}{|l|}{$22: 5(4 Z, 7 Z, 10 Z, 13 Z, 16 Z)$} \\
\hline \multicolumn{3}{|l|}{$22: 5(7 Z, 10 Z, 13 Z, 16 Z, 19 Z)$} \\
\hline $22: 6(4 Z, 7 Z, 10 Z, 13 Z, 16 Z, 19 Z)$ & & \\
\hline
\end{tabular}

For the marked (*) FAMEs the double bond positions were only tentatively assigned.

\subsection{Distribution of four important PUFAs among strains of the SAG algal culture collection}

The distribution patterns of FAs among and within the 17 groups (phyla or classes) of microalgae and the cyanobacteria comprised by the examined strains was investigated in more detail for four PUFAs which are of high nutritional interest (Table 3 ). The frequency of occurrence of these four PUFAs in a certain group of microalgae is given as the percentage of strains with a certain FA from all examined strains in Table 3.

Because the SAG culture collection focuses on microscopic algae from terrestrial habitats, the Haptophyta, Dinophyta and Phaeophyceae were just poorly represented. Therefore, the recovered distribution patterns in 
Table 2 Comparison of the major FA composition of algae observed in this study against data published previously

\begin{tabular}{|c|c|c|c|c|c|c|c|c|c|c|c|c|c|c|c|}
\hline \multirow[t]{2}{*}{ Species } & \multirow{2}{*}{$\frac{\mathrm{FA}}{14: 0}$} & \multirow{2}{*}{$\begin{array}{l}\% \\
16: 0 \\
\end{array}$} & \multirow{2}{*}{$\begin{array}{l}\text { of } \\
16: 1\end{array}$} & \multicolumn{11}{|l|}{ total) } & \multirow[t]{2}{*}{ Ref } \\
\hline & & & & $16: 2$ & $16: 3$ & $16: 4$ & 18:0 & $18: 1$ & $18: 2$ & $18: 3$ & $18: 4$ & $20: 4$ & $20: 5$ & $22: 6$ & \\
\hline \multicolumn{16}{|l|}{ Bacillariophyceae } \\
\hline Phaeodactylum & 9.2 & 26.8 & 45.4 & - & - & - & 0.7 & 4.6 & - & - & - & - & 12.3 & 1.1 & a \\
\hline \multirow[t]{2}{*}{ tricornutum } & 9.4 & 23.7 & 35.8 & - & - & - & 6.0 & 3.3 & 4.4 & 3.2 & 0.2 & - & 13.3 & 0.9 & $b$ \\
\hline & 6.7 & 14.7 & 43.6 & 2.0 & - & - & - & 15.8 & 0.5 & 0.4 & 1.1 & - & 14.4 & 0.7 & e \\
\hline \multirow[t]{2}{*}{ Thalassiosira weissflogii } & 25.9 & 28.8 & 28.7 & - & - & 7.4 & 1.5 & 3.3 & - & 0.3 & - & - & 4.0 & 0.1 & $\mathrm{~b}$ \\
\hline & 8.8 & 36.6 & 40.5 & - & - & - & - & 14.0 & - & - & - & - & - & - & e \\
\hline \multicolumn{16}{|l|}{ Chlorophyceae } \\
\hline \multirow[t]{2}{*}{ Dunaliella primolecta } & 0.4 & 21.8 & 4.5 & 0.9 & 2.5 & 12.3 & 0.8 & 6.4 & 6.2 & 41.1 & 4.1 & - & - & - & $\mathrm{b}$ \\
\hline & 0.6 & 26.0 & 0.9 & - & - & - & 1.6 & 16.3 & 7.0 & 38.7 & 0.6 & - & - & - & e \\
\hline \multirow[t]{2}{*}{ Nannochloris sp. } & 1.8 & 15.1 & 16.6 & - & 0.2 & - & 1.0 & 57.7 & 0.6 & 0.8 & 0.3 & 5.9 & - & - & $b$ \\
\hline & 13.3 & 17.8 & - & - & - & - & - & 23.9 & 10.8 & 28.2 & 6.1 & - & - & - & e \\
\hline \multirow[t]{2}{*}{ Parietochloris incisa } & - & 10.0 & 2.0 & 1.0 & 1.0 & - & 3.0 & 16.0 & 17.0 & 3.0 & - & 46.0 & 1.0 & - & c \\
\hline & 0 & 19.8 & - & 5.2 & - & - & 18.2 & 10.2 & 14.3 & 14.3 & - & 14.0 & 4.3 & - & e \\
\hline \multicolumn{16}{|l|}{ Cyanophyceae } \\
\hline \multirow[t]{2}{*}{ Nostoc commune } & 0.3 & 43.5 & 11.3 & 0.4 & - & - & 1.5 & 6.9 & 19.3 & 16.3 & - & - & - & - & $d$ \\
\hline & - & 25.3 & 24.1 & - & - & - & - & - & 12.5 & 38.1 & - & - & - & - & e \\
\hline \multirow[t]{2}{*}{ Synechocystis sp. } & 13.4 & 26.5 & 43.6 & - & - & - & 3.5 & 8.0 & 0.2 & 4.7 & - & - & - & - & $b$ \\
\hline & 42.5 & 18.8 & 30.1 & - & - & - & - & - & - & 14.2 & - & - & - & - & e \\
\hline \multicolumn{16}{|l|}{ Haptophyceae } \\
\hline \multirow[t]{2}{*}{ Pavlova lutheri } & 11.8 & 23.6 & 28.3 & - & - & - & 2.0 & 12.4 & - & - & - & - & 12.1 & 9.7 & a \\
\hline & 10.1 & 11.1 & 26.3 & - & - & - & - & 5.2 & 0.6 & 0.5 & 9.1 & 0.3 & 18.0 & 9.7 & e \\
\hline \multicolumn{16}{|l|}{ Prymnesiophyceae } \\
\hline \multirow[t]{2}{*}{ Emiliana huxleyi } & 41.7 & 17.7 & 5.5 & - & - & - & 2.1 & 21.7 & 0.9 & 5.5 & 5.0 & - & - & - & $b$ \\
\hline & 18.8 & 10.3 & - & - & - & - & 10.8 & 42.2 & - & - & 8.7 & - & - & 9.2 & e \\
\hline \multicolumn{16}{|l|}{ Raphidophyceae } \\
\hline \multirow[t]{2}{*}{ Heterosigma akashiwo } & 6.2 & 46.3 & 21.3 & - & - & 0.4 & 0.5 & 2.7 & 1.6 & 4.2 & 7.3 & - & 8.7 & 0.7 & $b$ \\
\hline & 6.6 & 40.0 & 12.7 & 4.0 & - & - & - & - & 4.5 & 6.7 & 5.2 & 3.5 & 14.8 & - & e \\
\hline
\end{tabular}

a [3]

b [4]

c [20]

d [12]

e this work

these and other poorly represented groups may not be representative for the whole group. For instance, for Phaeophyceae mainly microscopic forms (e.g., Ectocarpus and the freshwater genus Bodanella) were available and the examined Rhodophyta strains covered mostly freshwater forms or those from terrestrial habitats (e.g., Porphyridium). Although diatoms are very diverse in terrestrial habitats, the examined small sample of available diatom strains (18) does by far not adequately represent this group which is probably the most speciesrich algal group. Also, for each of the two classes of Stramenopiles (heterokont algae), Phaeothamniophyceae and Raphidophyceae, just two strains are maintained at the SAG and, therefore, are not further discussed here. Similarly, there is only a single strain of Chlorarachniophyta (Rhizaria supergroup) in the SAG.

The very long chain PUFA docosahexaenoic acid (DHA, 22:6(4Z, 7Z, 10Z, 13Z, 16Z, 19Z)) was the third most frequent FA, present in 15 out of 20 examined groups (Table 3). In the Dinophyta, Haptophyta and Euglenoids DHA-containing strains were particularly frequent and DHA was found there in relatively high percentages of total FA content, i.e. in $60 \%$ or more of these strains the DHA proportion was higher than 5\%. In the single studied dinophyte strain of Ceratium horridum the DHA proportion was even $29.3 \%$. In the other groups DHA was found in rather low frequencies and also mostly in rather small proportions, i.e. less than $1 \%$ of total FA content. Although DHA was found in the Cryptophyta and Bacillariophyceae in about every fifth strain, its percentage of total FA content was less than $5 \%$ there, except in Cryptomonas baltica SAG 18.80 (Cryptophyta) where it is was $13.7 \%$. Despite DHA was found in rather low frequencies in the green algae (Chlorophyta), the second highest DHA content of all SAG strains, $18.9 \%$ of total FA, was found in the chlorophyte Chlorococcum 


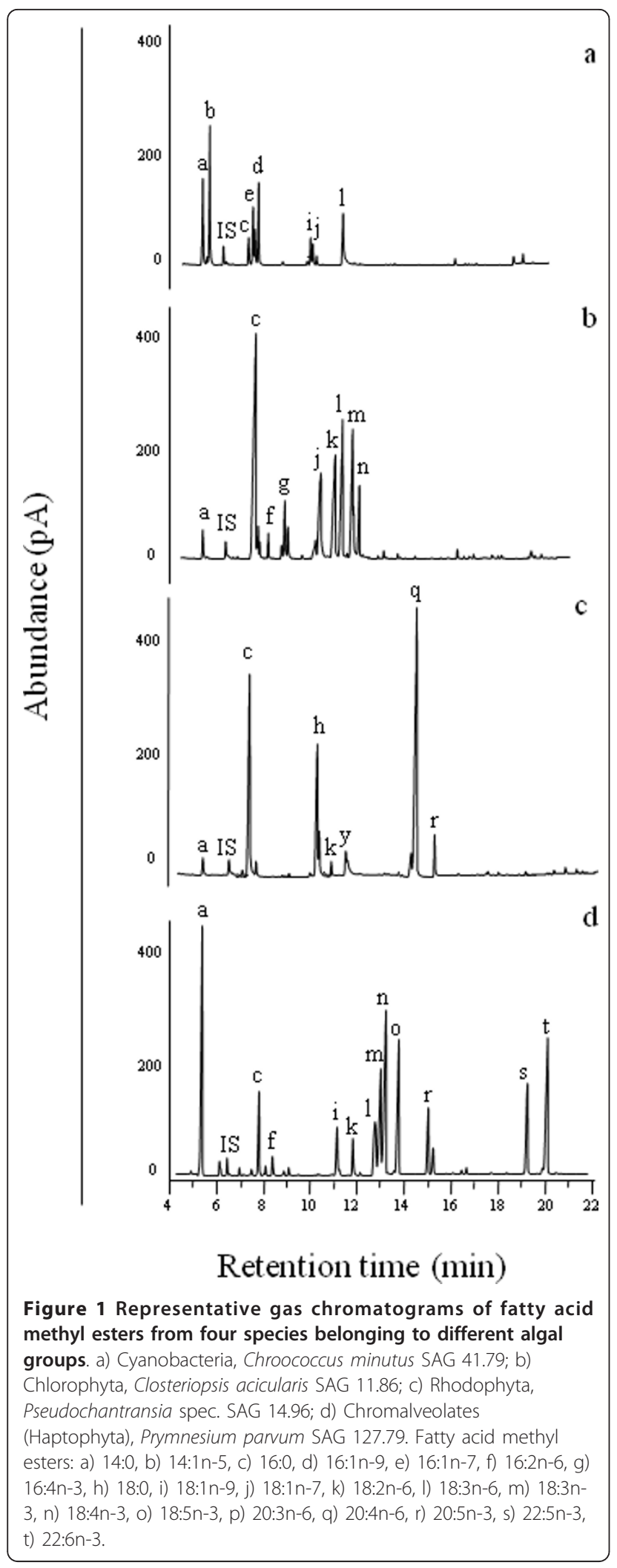

novae-angliae SAG 5.85 , followed by the trebouxiophyte Prototheca zopfii SAG 263-8 with 14.2\%. Together these findings are in accordance with DHA amounts described before for specific groups of alga $[3,4,14,15]$.

Eicosapentaenoic acid (EPA, 20:5(5Z, 8Z, 11Z, 14Z, $17 Z)$ ) was one of the most common PUFAs, found in all of the 17 groups covered by our study (Table 3). EPAcontaining strains were particularly frequent in the Eustigmatophyceae, Glaucophyta, Xanthophyceae and Rhodophyta. The highest EPA proportions of total FA content were in the Rhodophyta, with about $81 \%$ of the strains exhibiting more than 10\% EPA. The highest values were $52.4 \%$ in Compsopogonopsis leptoclados SAG 106.79 and $44.9 \%$ in Acrochaetium virgatulum SAG 1.81. Also strains of three species of Porphyridium contained high amounts of EPA (31.2\% in P. sordidum SAG O 500, 27.5\% in P. aerugineum SAG $110.79,26.7 \%$ in $P$. purpureum SAG 1380-1a). This is in agreement with a report on $P$. cruentum suggesting that red algae are a rich source of EPA [16]. Despite EPA was rather frequently found in the Glaucophyta, only about half of all strains had EPA proportions greater than 10\% (maximum 31.1\% in Glaucocystis nostochinearum SAG 28.80). This is in agreement with another study which showed high amounts of EPA (besides ARA) in the glaucophyte Cyanophora paradoxa [17]. The highest percentage (87\%) of strains with an EPA proportion of greater than $10 \%$ was in the Dinophyta, but with a maximum of just 24.3\% in Pyrocystis lunula SAG 2014. In the Euglenoids, Xanthophyceae and Eustigmatophyceae about $67 \%$ of all strains had an EPA proportion of greater than $10 \%$ with maximum values of about $31 \%$ (31.4\% in Heterococcus fuornensis SAG 835-5, 31.6\% in Euglena proxima SAG 1224-11a) and 34.6\% in Goniochloris sculpta SAG 29.96. EPA was rarely found and mostly in insignificant amounts $(<5 \%)$ in most green algae, but three strains had an exceptionally higher content of about $20 \%$ of total FAs (24.2\%, Chlorella sp. SAG 242.80; 24.0\%, Chlamydomonas allensworthii SAG 28.98; 22.3\%, Cylindrocapsa involuta SAG 314-1). EPA was the only FA recovered from Chlorarachnion repens SAG 26.97 (Chlorarachniophyta). That Xanthophyceae and Eustigmatophyceae contain EPA in relatively high proportions while green algae rarely accumulate EPA supports previous studies $[3,4,14,15,18]$.

Arachidonic acid (ARA, 20:4(5Z, 8Z, 11Z, 14Z)) was most frequently found in the Phaeophyceae where it was present in all strains except one investigated strain (Table 3); in about $54 \%$ of all Phaeophyceae strains the proportion of ARA was higher than $10 \%$, but with a maximum of just $17.7 \%$ in Halopteris filicina SAG 
Table 3 Frequency of four selected PUFAs in 17 taxonomic groups of microalgae on which the examined 2071 strains of the SAG culture collection were distributed, and the size of each group (in total number of strains)

\begin{tabular}{|c|c|c|c|c|c|c|c|}
\hline & & & no. of strains & DHA & EPA & ARA & GLA \\
\hline Cyanobacteria & & & 223 & 1.3 & 0.9 & 0.4 & 12.1 \\
\hline \multirow[t]{7}{*}{ Plantae } & Glaucophyta & & 15 & & 80.0 & 46.7 & 6.7 \\
\hline & Chlorophyta & Chlorophyceae & 927 & 5.1 & 6.9 & 5.7 & 26.2 \\
\hline & & Trebouxiophyceae & 253 & 4.3 & 16.6 & 22.9 & 6.3 \\
\hline & & Ulvophyceae & 70 & 4.3 & 22.9 & 12.9 & 7.1 \\
\hline & & prasinophytes & 21 & 14.3 & 33.3 & 42.9 & 57.1 \\
\hline & Charophyta & & 159 & 1.3 & 17.6 & 13.8 & 31.4 \\
\hline & Rhodophyta & & 78 & & 70.5 & 67.9 & 3.8 \\
\hline Excavates & Euglenoids & & 131 & 42.7 & 44.3 & 51.1 & \\
\hline \multirow[t]{9}{*}{ Chromalveolates } & Stramenopiles & Bacillariophyceae & 18 & 22.2 & 44.4 & 11.1 & 11.1 \\
\hline & & Xanthophyceae & 81 & 4.9 & 75.3 & 49.4 & 16.1 \\
\hline & & Eustigmatophyceae & 17 & & 88.2 & 41.2 & 5.9 \\
\hline & & Phaeophyceae & 12 & & 58.3 & 91.7 & 16.7 \\
\hline & & Chryso-/Synurophyceae & 12 & 16.7 & 33.3 & 8.3 & 16.7 \\
\hline & Haptophyta & & 13 & 84.6 & 61.5 & 7.7 & \\
\hline & Cryptophyta & Cryptophyta & 27 & 22.2 & 66.7 & 3.7 & 3.7 \\
\hline & Alveolates & Dinophyta & 14 & 64.3 & 57.1 & 14.3 & \\
\hline & & & 2071 & & & & \\
\hline
\end{tabular}

The frequency of PUFAs is shown as the percentage of the total number of strains examined per group.

10.96. ARA had the highest proportion of total FA in the Rhodophyta; there even about $77 \%$ of all strains had an ARA content of more than $10 \%$ with a maximum of 68.3\% in Pseudochantransia sp. SAG 19.96. Interestingly, the ARA content was rather high but variable among the eight examined multiple isolates of the rhodophyte Porphyridium purpureum. While the average ARA proportion was about $31 \%$ in six strains, it was just $3.8 \%$ in SAG $1380-1$ d, but $44.5 \%$ in SAG 1380-1e. We have no explanation for this variation yet; both strains were isolated from marine habitats and are kept under the same culture conditions. High proportions of ARA (as well as EPA) were already found characteristic of another species of Porphyridium cruentum [16]. ARA was present in about half of all investigated Euglenoid strains and with relatively high proportions of total FA content, i.e. about one third of the strains exhibited more than 5\% ARA with extraordinarily high values of $41.3 \%$ and $34.3 \%$ in Rhabdomonas incurva SAG 1271-8 and Khawkinea quartana SAG 1204-9. Interestingly, another strain of the same species $K$. quartana, SAG 1204-9, had less than half (13.3\%) of ARA content and in five other species of Rhabdomonas no ARA was detected. This demonstrates that FA contents may be rather variable between species of the same genus and even among multiple isolates of the same species. Although about half of all examined strains for the Xanthophyceae and Eustigmatophyceae contained ARA (Table 3), they had this FA in relatively low proportions. Only one fourth of the ARA-containing Xanthophyceae strains exhibited more than $5 \%$ and in the Eustigmatophyceae even no strain reached $5 \%$. ARA was rarely found in the green algae, i.e. with an average frequency of about $14 \%$ in the phyla Chlorophyta and Streptophyta, except for prasinophyte green algae where ARA was present in $42.9 \%$ of all strains (Table 3). However, there were a few single green algal examples with extraordinarily high ARA contents, i.e. $73.8 \%$ (corresponding to $102 \mu \mathrm{g} / \mathrm{mg}$ of dry weight, the highest ARA content detected in all investigated SAG strains) in the chlorophyte Palmodictyon varium SAG 3.92, followed by $52.9 \%$ in the chlorophyte Trochisciopsis tetraspora SAG 19.95 and $51.8 \%$ in the trebouxiophyte Myrmecia bisecta SAG 2043. That a high ARA content was found in the latter strain is in agreement with that it has been found a close relative with Parietochloris incisa (syn. Lobosphaeropsis incisa, Myrmecia incisa) [19]. P. incisa has been assigned an "oleaginous microalga" and the richest plant source of ARA known so far due to its capability to accumulate high amounts of ARA (up to 59\% of its total FA content) [20]. Interestingly, the SAG strain of P. incisa (Lobosphaera incisa SAG 2007) had with $13.2 \%$ a much lower ARA content (Table 2).

$\gamma$-Linolenic acid (GLA, 18:3(6Z, 9Z, 12Z)) was the third most common FA in the studied sample of SAG microalgal strains, missing only in the Haptophyta, Dinophyta and Euglenoids (Table 3). It was most frequently detected in two lineages of green algae, the prasinophytes and the Streptophyta. In prasinophytes, however, GLA was present only in one out of five genera available for that 
group, Tetraselmis, and there in 12 out of the 17 available strains and with variable proportions, i.e. $0.5-7.3 \%$ of total FA content. In the Streptophyta, GLA was more widely distributed, i.e. it was detected in 17 out of 41 examined genera. GLA distribution was rather variable within strains and species of a certain streptophyte genus, similar to findings of ARA in other genera. Relatively high percentages of GLA were found in species/ strains of Closterium (16.5\% in C. baillyanum SAG 50.89, $8 \%$ in C. lunula SAG 7.84), but GLA was not found in the other 12 strains of that genus. Similarly, in the many strains available for Cosmarium (25) and Micrasterias (16), GLA was found in only 11 and 2 strains, respectively. The highest percentages of GLA were found in the green algal class Chlorophyceae (29.9\% in Deasonia multinucleata SAG 25.95, 28.5\% in Desmodesmus multiformis SAG 26.91) and in Cyanobacteria (24.8\% in Spirulina maxima SAG 84.79 ). In about one third (32\%) of all chlorophyte GLA strains this FA had precentages of 5\% and higher. Distribution of GLA in the cyanobacteria was rather patchy, i.e. the 27 cyanobacteria strains with GLA were mainly restricted to three genera, Calothrix (8 strains), Microcystis (7 strains) and Spirulina (6 strains). Also within each of these genera the GLA percentages were quite variable, e.g. in Spirulina it varied from $4.6 \%$ to $24.8 \%$, and three strains where without GLA. FA composition has previously been used to discriminate cyanobacteria in isolates and natural samples at the generic level $[21,22]$. To discriminate species of cyanobacteria, as an additional marker the hydrocarbon composition was used in an earlier study, but in our study we failed to detect any substance out of this group [23]. Interestingly, GLA was the only FA that was detected in more than three out of the 223 examined strains. Therefore, the SAG cyanobacteria strains may be roughly divided into those with GLA present (few genera) and those where almost no PUFAs were present. This corresponds to the earlier findings that described a bipartition of cyanobacteria, independent of their taxonomic position, into genera producing $\mathrm{C}-18$ PUFA and those which do not [24,25].

The prasinophyte genus Tetraselmis presented an interesting example to test for FA variation among closely related isolates. Nine strains assigned to that genus have been isolated from the same (marine) locality and regarded as the same species by the isolator (U.G. Schlösser, pers. comm.). Only in two strains DHA was present, but in very small traces $(0.3 \%$ and $0.4 \%)$. In contrast, ARA and GLA were found in all isolates with percentages varying from $0.8 \%$ to $2.7 \%$ and $0.5 \%$ to $7.3 \%$, respectively.

\subsection{Analysis of FA distribution patterns}

The detected fatty acid (FA) composition of the 2076 investigated strains was statistically analyzed to test whether certain patterns of FA distribution among the various investigated algal groups are present that may correspond to their phylogenetic relationships. In a first set of three analyses (higher taxonomic levels) it was tested 1) whether FA distribution patterns may reflect differences among algal phyla derived from primary (Plantae supergroup) or secondary endocytobiosis (Chromalveolates, Euglenoids) compared to cyanobacteria representing the plastid origin, 2) the distinction of phyla within the Plantae supergroup (Chlorophyta, Streptophyta, Rhodophyta/Glaucophyta) and 3) major evolutionary lineages (classes) within the Chlorophyta. A second set of analyses focused at the generic level, i.e.it was tested whether separation of genera as based on previous $18 \mathrm{~S}$ rDNA sequence analyses suggested for Chlamydomonas s.l., Chlorella s.l. and Scenedesmus s.l. are reflected in the FA distribution patterns. For the first set of analyses the many species (266) which were represented as multiple strains (e.g., Chlamydomonas moewusii, 28) had to be reduced to only a single strain per species to avoid biases. This included also the multiple strains unidentified at the species level, i.e. labelled with "sp." instead a species name (e.g., Chlorogonium sp., 26). The SAG's Chlorophyta strains were particularly rich in such multiple strains. Also excluded were those strains where only a single FA was detected. This reduced the total number of strains considered in our calculations to 1193 . The strains were then divided into eleven groups roughly corresponding to phyla or classes (Additional file 2). Strains belonging to the Chlorophyta (61\% of all investigated strains) were further subdivided into the three classes, Chlorophyceae, Trebouxiophyceae, and Ulvophyceae, whereas the prasinophyte SAG green algal strains $(1.7 \%$ of all considered Chlorophyta strains) were excluded from the analyses because they comprised only very few species (10). The strains of Glaucophyta (15) and Rhodophyta (81) were collectively treated as one composite unit. The Rhizaria - Chlorarachniophyta, was represented just by a single strain and, thus, was omitted from the statistical analyses.

Higher taxonomic levels analyses It was tested whether distribution patterns of FA composition on the investigated strains delineate the three "super groups" of eukaryotic algae, Plantae, Chromalveolates and Excavates (Euglenoids), and the cyanobacteria from each other. The Plantae super group comprises exclusively eukaryotes with plastids derived from primary endocytobiosis, i.e. a cyanobacterium was transformed into an organelle through uptake and retention by the host cell followed by the loss of much of its genome [26]. Chromalveolate algae as well as the Euglenoids (the only algal lineage of Excavates) acquired their plastids through secondary endocytobiosis from rhodophyte and a green alga, respectively [26,27]. To consider almost 
equal numbers of strains for all four groups, 100 strains of Plantae, Chromalveolates and Cyanobacteria were randomly selected which closely amounts the total number of considered euglenoid strains (73). The ordination which resulted from CVA (Canonical Variates Analysis, multigroup discriminant analysis) pointed out a strong difference between cyanobacteria/primary endocytobiosis (Plantae) and the two groups representing secondary endocytobiosis (Chromalveolates/Euglenoids) (Figure 2). The observed difference was without exception supported by non-parametric significance tests for multidimensional data (NP-MANOVA and ANOSIM). Following SIMPER, the lowest observed dissimilarity (63.55\%) was between Cyanobacteria and Plantae, while the highest (77.29\%) was between Plantae and Chromalveolates. The first canonical variate (CV1) involved 99.99\% of all possible differences among the four groups, hence we examined for possible correlations between this axis and FAs. Four FAs were significantly and exclusively correlated with the first canonical variate

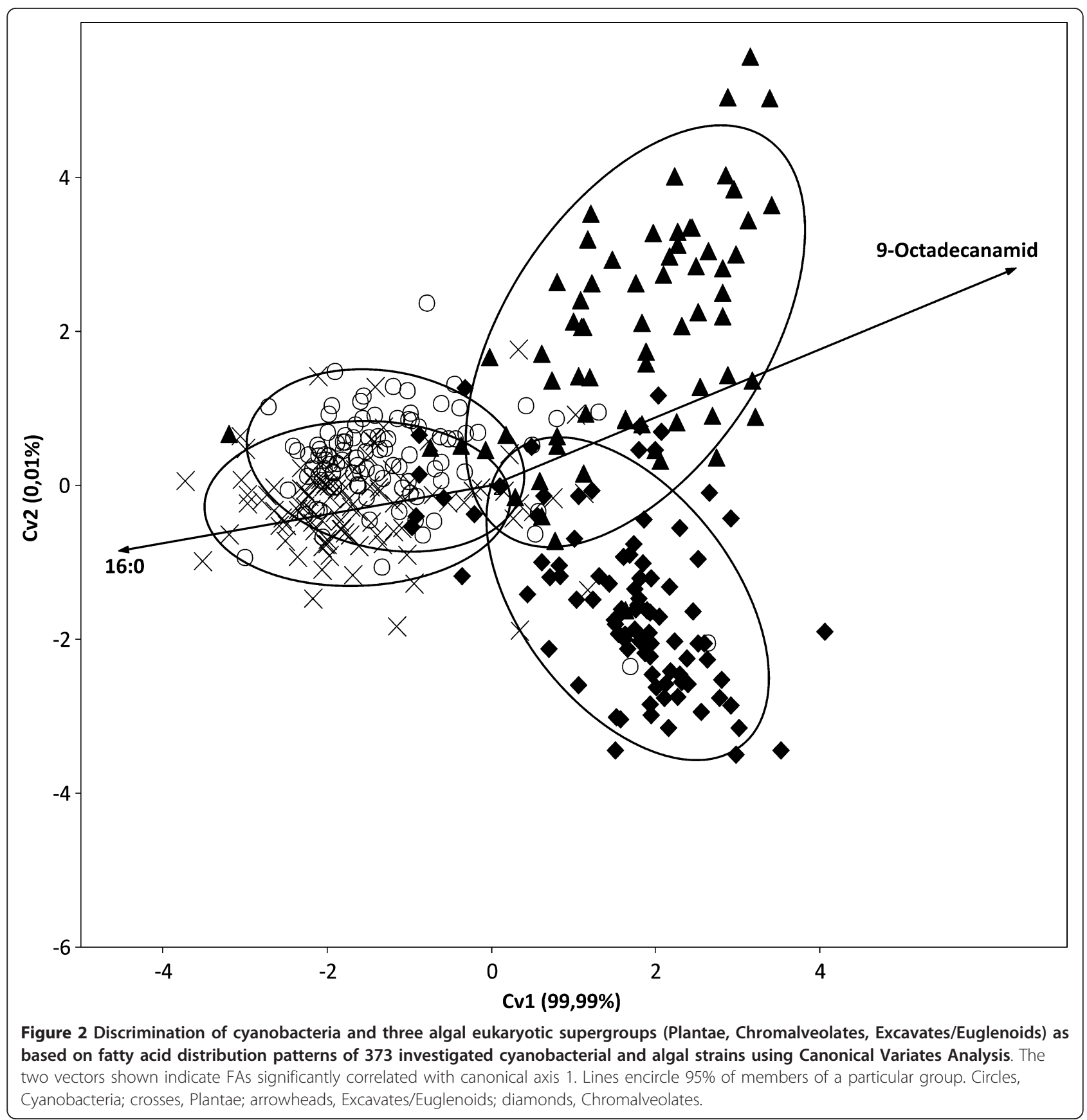


(CV1), i.e. $16: 0\left(\rho_{\mathrm{CV} 1}=-0.61 / \mathrm{p}<0.001\right), 18: 2(9 Z, 12 Z)$ $\left(\rho_{\mathrm{CV} 1}=-0.46 / \mathrm{p}<0.001\right), 9$-octadecanamid $\left(\rho_{\mathrm{CV} 1}=0.41 /\right.$ $\mathrm{p}<0.001)$, and $18: 1(9 Z)\left(\rho_{\mathrm{CV} 1}=-0.17 / \mathrm{p}=0.001\right)$. In a second analysis it was tested whether FA distribution patterns distinguish phyla of the Plantae super group, i.e. the two lineages of green algae, Chlorophyta and Streptophyta [28,29], and the composite Rhodophyta/ Glaucophyta group. Because the latter was with 54 strains the smallest group, it was compared with equally large random samples from each the Chlorophyta and Streptophyta (Table 3). The ordination diagram from a CVA of the total of 162 investigated strains clearly separated the Rhodophyta/Glaucophyta group from both green algal phyla (Figure 3). CV1 involved $79 \%$ of all possible differences and even CV2 was with $21 \%$ not negligible. The significance tests, NP-MANOVA and ANOSIM, supported the distinction of all three groups. SIMPER showed the Rhodophyta/Glaucophyta composite group

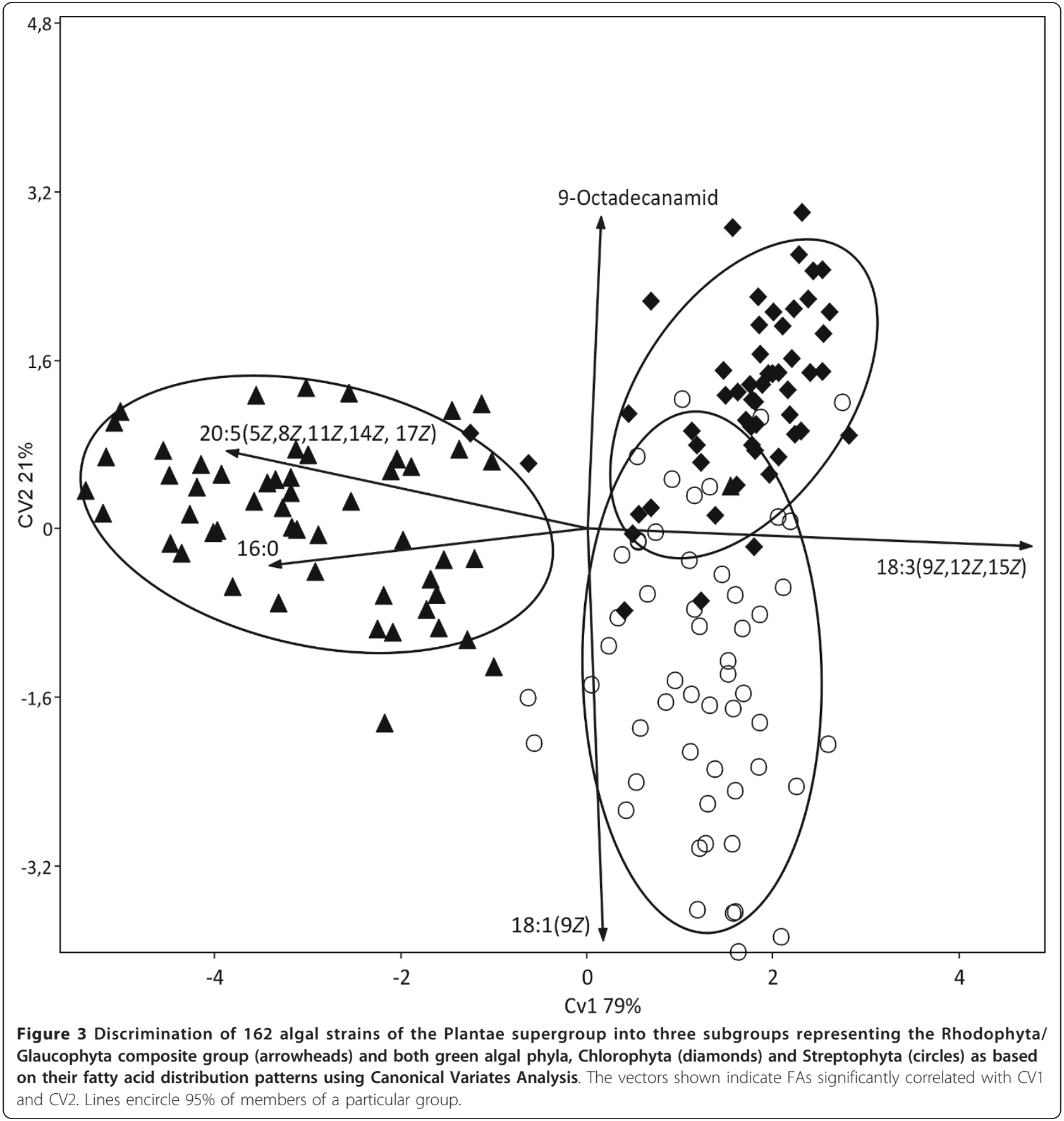


rather dissimilar from both green algal phyla, i.e. there were dissimilarities of $70.55 \%$ and $71.53 \%$ with the Chlorophyta and Streptophyta, respectively. The lowest dissimilarity (55.41\%) among the three tested groups was between Chlorophyta and Streptophyta. There were five FAs significantly and exclusively correlated with CV1, i.e. $18: 3(9 Z, 12 Z, 15 Z)\left(\rho_{\mathrm{CV} 1}=0.77 / \mathrm{p}<0.001\right), 20: 4\left(\rho_{\mathrm{CV} 1}=\right.$ $-0.49 / \mathrm{p}<0.001), 20: 5(5 Z, 8 Z, 11 Z, 14 Z, 17 Z)\left(\rho_{\mathrm{CV} 1}=\right.$ $-0.59 / \mathrm{p}<0.001), 18: 1(9 Z)\left(\rho_{\mathrm{CV} 1}=0.30 / \mathrm{p}=0.001\right)$ and $16: 0\left(\rho_{\mathrm{CV} 1}=-0.56 / \mathrm{p}=0,001\right)$. Two FAs were correlated exclusively with CV2, i.e. they discriminated Chlorophyta and Streptophyta, 18:1(9Z) $\left(\rho_{\mathrm{CV} 2}=-0.4477 / \mathrm{p}<0.001\right)$ and 9-octadecanamid $\left(\rho_{\mathrm{CV} 2}=0.34 / \mathrm{p}<0.001\right)$. The by far largest fraction of all considered strains $(60.3 \%)$ were from the Chlorophyta which made it interesting to test whether FA distribution patterns can discriminate between the three classes of Chlorophyta, the Chlorophyceae, Trebouxiophyceae and Ulvophyceae. Ulvophyceae was the smallest of the three with just 49 strains and, therefore, random samples of almost the same size (54) from each of the other two classes were used for the statistical analyses. The CVA did not reveal any distinct groups, i.e. the analyzed strains tended to form three groups corresponding to the three green algal classes, but with a considerable overlap among them (Figure 4). However, the three classes were found significantly distinct from each other in both employed significance tests and SIMPER. The latter and correlation analyses allowed to consider 9-octadecanamid $\left(\rho_{\mathrm{CV} 1}=-0.58 / \mathrm{p}<0.001\right.$; $\left.\rho_{\mathrm{CV} 2}=-0.22 / \mathrm{p}<0.010\right)$ and the FA $18: 2(9 Z, 12 Z)\left(\rho_{\mathrm{CV} 1}=\right.$ $\left.-0.44 / \mathrm{p}<0.001 ; \rho_{\mathrm{CV} 2}=-0.53 / \mathrm{p}<0.001\right)$ as the only variables to discriminate well Ulvophyceae from Chlorophyceae/Trebouxiophyceae and Trebouxiophyceae from Ulvophyceae/Chlorophyceae, respectively.

Generic level analyses The three previous analyses showed that phylogenetic relationships at the level of phyla and classes among algal groups were reflected in FA distribution patterns using a large sample of strains. Therefore, in a second group of analyses, we tested whether differences in FA distribution patterns may resolve the same distinction of genera as in rRNA gene sequence analyses. To test this, we selected three genera which are widely used in biotechnological applications and well represented by SAG strains, i.e. Chlorella s.l., Scenedesmus s.l. and Chlamydomonas s.l.. Recent18S rRNA gene sequence analyses revealed each of the three as paraor polyphyletic assemblages encompassing several distinct genera. For Chlamydomonas we selected 17 species (53 strains), out of which 9 were represented by multiple strains (e.g., C. reinhardtii, 16), which were distributed on five independent lineages/clades (= genera) in the $18 \mathrm{~S}$ rDNA phylogeny [30]. To better represent the "Oogamochlamys" clade also two strains from the UTEX collection
$(2213,1753)$ were included. The NMDS ordination clearly separated the members of the "Reinhardtii" clade (upper right in Figure 5), except for three strains, from those of the "Chloromonas" clade (lower left in Figure 5). However, the "Chloromonas" group as revealed by the FA patterns also included the three investigated strains of the "Moewusii" and four of the "Oogamochlamys" clades which was in contrast to the $18 \mathrm{~S}$ rDNA phylogenies of [30]. Also in contrast to the rDNA phylogenies, the FA analyses split the genus Lobochlamys, i.e. L. culleus was part of the "Chloromonas" group while L. segnis belonged to the "Reinhardtii" group. Strains of Oogamochlamys were also separated on both FA groups, in contrast to their species assignments as based on the $18 \mathrm{~S}$ rDNA analyses.

Species and strains formerly assigned to a single genus Scenedesmus were shown to be actually distributed on several genera by rRNA gene sequence analyses. For example, the genus Acutodesmus has been segregated from Scenedesmus [31,32]. A NMDS ordination plot of FA distribution patterns revealed a tendency among the studied strains to be distributed on two clusters, i.e. one cluster of 8 strains of Acutodesmus (mainly including multiple strains of $A$. obliquus) was clearly separated from another cluster containing mainly strains of Scenedesmus s.str. (Figure 6). The multiple strains of S. vacuolatus were grouped together with four other strains of the genus, except for SAG 211-11n which was close to the Acutodesmus cluster. The multiple strains of $A$. obliquus, however, were distributed on both clusters (Figure 6). Seven strains of A. obliquus mainly formed up the Acutodesmus cluster, whereas five other A. obliquus strains grouped together with strains of Scenedesmus s.str. This means that within the same green algal species, $A$. obliquus, two distinct FA patterns exist. AFLP fingerprints already showed extensive genetic variation among the multiple strains of $A$. obliquus while ITS2 rDNA sequence comparisons demonstrated conspecificity of the multiple strains, except for SAG 27620 (T. Friedl, unpubl. observation). Therefore, the finding of A. obliquus strains being separated in two FA pattern groups favours the view that genetic differences resolved by AFLPs may correspond to different phenotypic properties. Consequently, it may be crucial to carefully record which strain has been used in any application [33]. Though strain SAG 276-20 was found not to belong to the same species, $A$. obliquus, its FA pattern suggests that it may still be a member of Acutodesmus because it was grouped in the Acutodesmus cluster (Figure 6).

Chlorella vulgaris forms another example where extensive genetic variation among multiple strains of the same species has been detected by AFLP analyses [33]. The 15 multiple SAG strains of C. vulgaris were compared to 19 other Chlorella and Chlorella-like strains, i.e. their closest relatives as seen in $18 \mathrm{~S}$ rDNA phylogenies, C. sorokiniana 


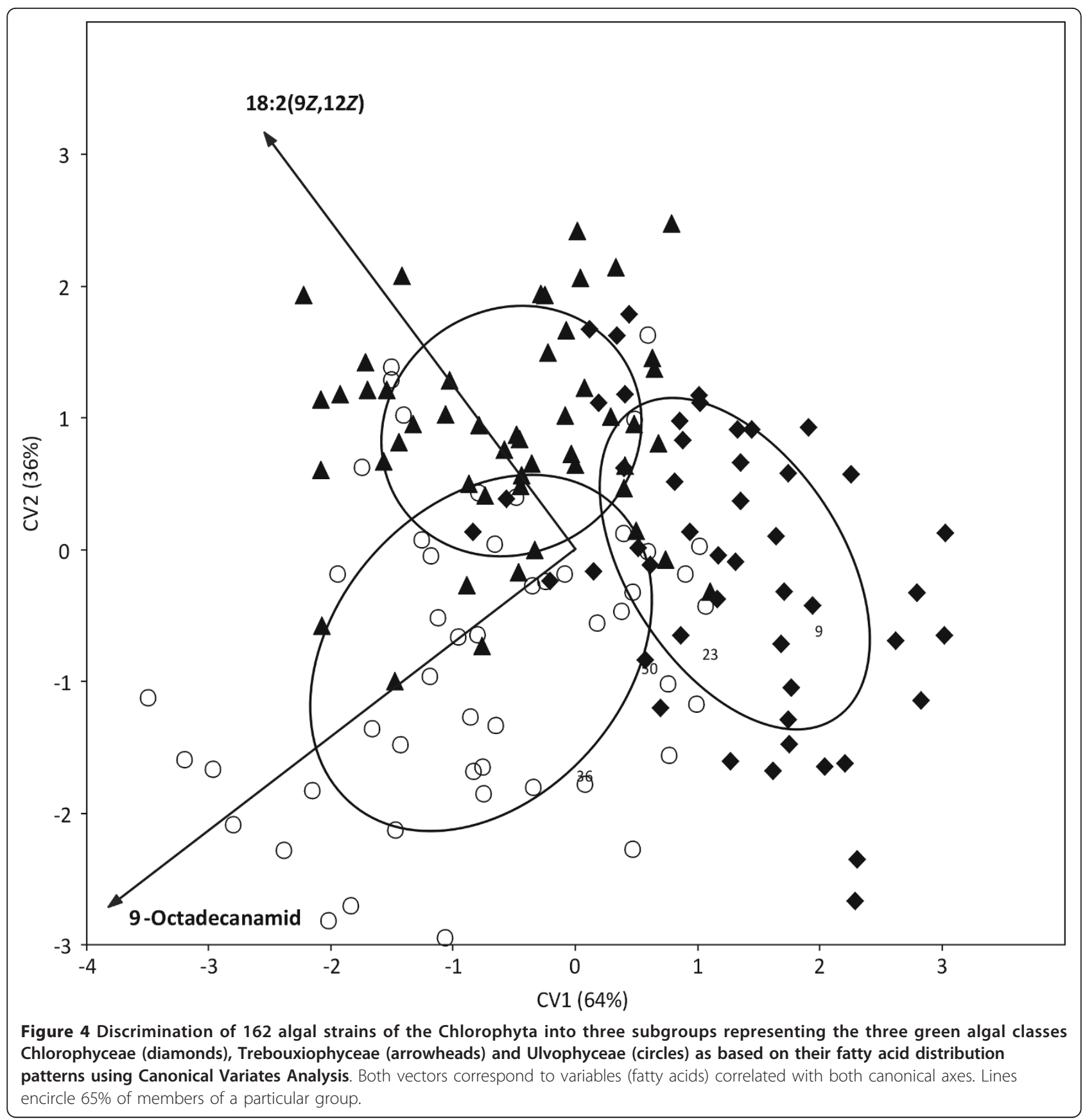

and C. lobophora, members of the Parachlorella clade sensu [34] as well as more distantly related strains, i.e. from the Watanabea and Prasiola clades sensu [35]. NMDS ordination based on FA distribution pattern showed almost no variation within the multiple strains of C. vulgaris and clustered them together, except for strain SAG 211-1e (Figure 7). Another cluster distant from $C$. vulgaris was formed by members of the Watanabeaclade, whereas Chlorella-like algae of the Prasiola-clade were not clustered together.

\section{Conclusion}

The algae collection at the SAG represents a valuable resource of natural products as shown in the present study for FAs and other hydrophobic metabolites. Several general trends in FA distribution reflect phylogenetic relationships among phyla and classes as seen in genomic and molecular phylogenies and this makes FA distribution patterns an additional feature to define taxa of higher rank in algae. However the FA profile alone may be no useful marker to distinguish among different 


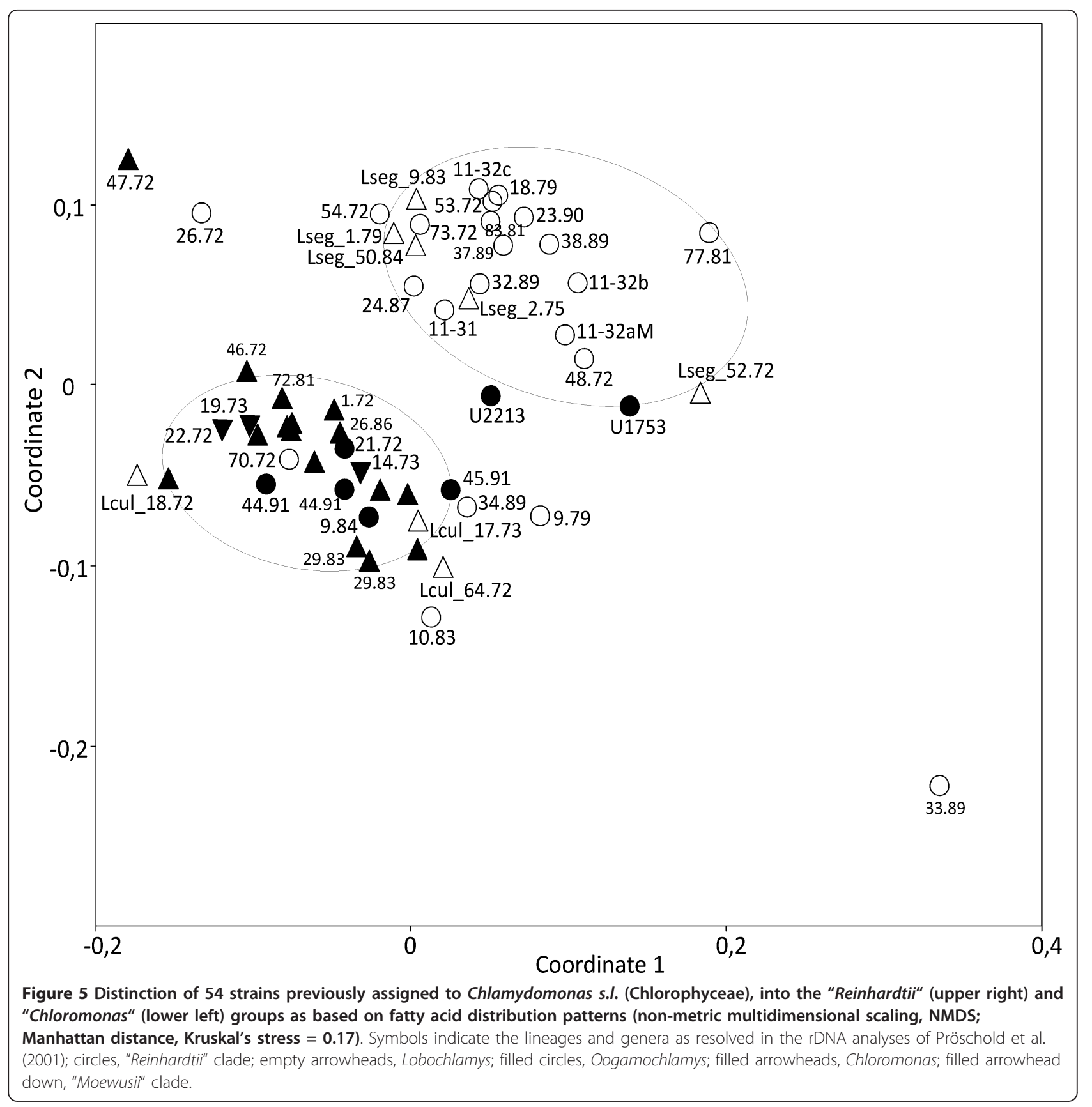

genera and species. For this, the comparison of further metabolites, like sterols, entire lipids and hydrocarbons should be considered. Thus, PUFA contents in microalgae are rather difficult to predict at the levels of genera and species, making it difficult to select appropriate strains for biotechnological research/applications which aim at yielding high lipid contents. Therefore, each additional or novel isolate will be worth of examination for its PUFA content.

\section{Methods}

Preparation of microalgal cultures

The microalgal cells were harvested from cultures at the stationary phase and stored at $-20^{\circ} \mathrm{C}$. Stationary phase was reached after different periods of culturing ranging from three months to about one year, depending on the strain-specific SAG's standard maintenance protocols. Before FA extraction the algal material was lyophilised for two days until the cell pellets were totally dry. 


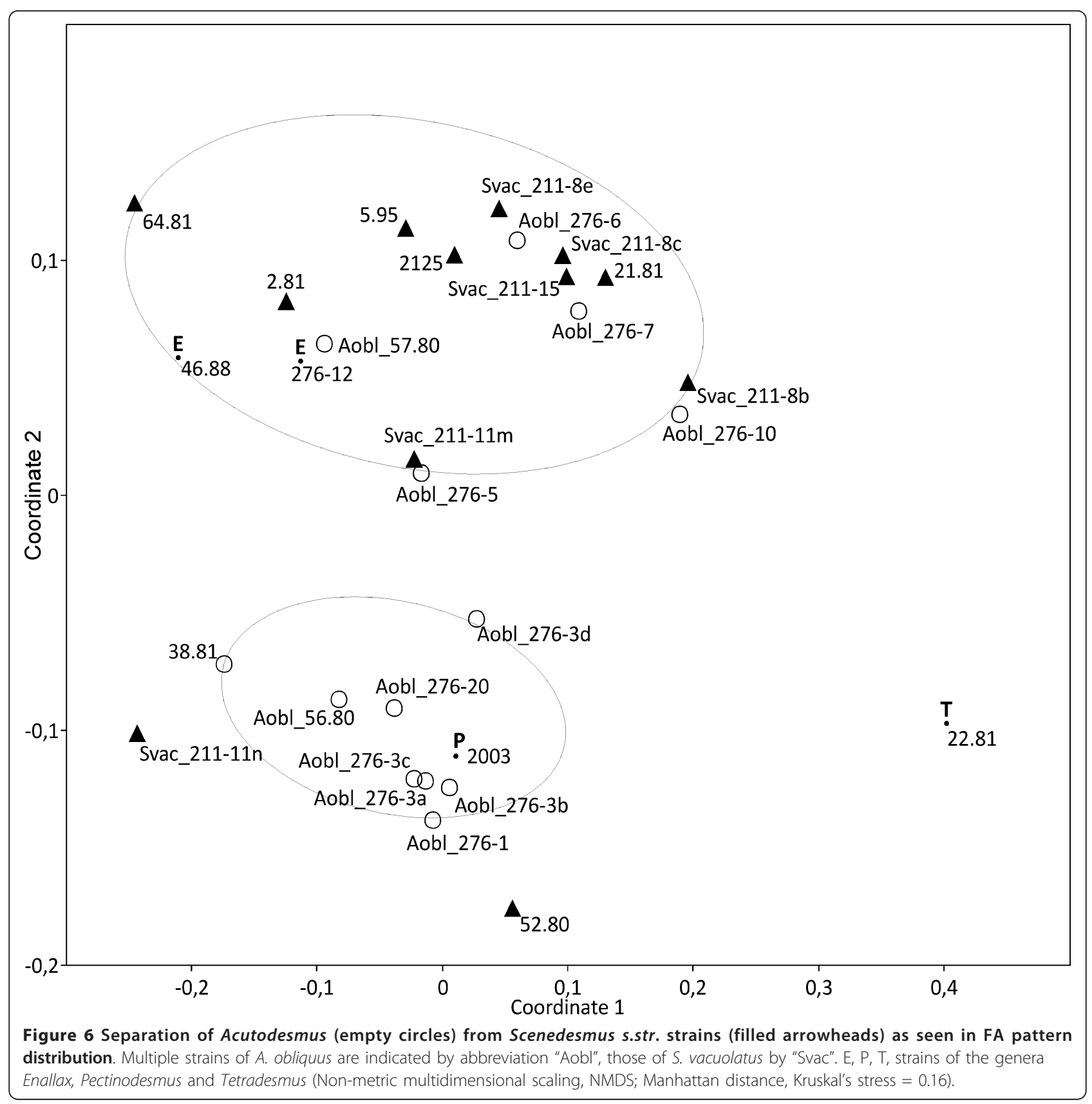

\section{Alkaline hydrolysis, transesterification and extraction of FA methyl esters (FAMEs)}

Prior to FAME extraction the dry weight of lyophilised algal material was determined and then the samples were transferred into a $2 \mathrm{ml}$ tube. The samples were extracted by adding $405 \mu \mathrm{l}$ of methanol/toluol 2:1 (v/v) followed by homogenisation of the cells with a potter (Heidolph RZR 2020, Schwabach) for $30 \mathrm{~s}$. To avoid autoxidation, the samples were overlaid with argon. As internal standard, $10 \mu \mathrm{g}$ of tripentadecanoate (diluted in $10 \mu \mathrm{l}$ toluol) was added. Transesterification of lipid bound FAs to their corresponding FAMEs was accomplished by adding $150 \mu \mathrm{l}$ sodium methoxide [36]. After $20 \mathrm{~min}$ shaking at RT the FAMEs were extracted two times with $500 \mu \mathrm{ln}$ hexane and $500 \mu \mathrm{l} 1 \mathrm{M} \mathrm{NaCl}$. The hexane phases were transferred into a $1.5 \mathrm{ml}$ tube and dried under streaming nitrogen. Finally the FAMEs were redissolved in $10 \mu \mathrm{l}$ acetonitrile and analysed by GC.

\section{Preparation of 4, 4-dimethyloxaline (DMOX) derivatives}

The position of double bonds of unknown FAME isomers was determined by analysing the corresponding 


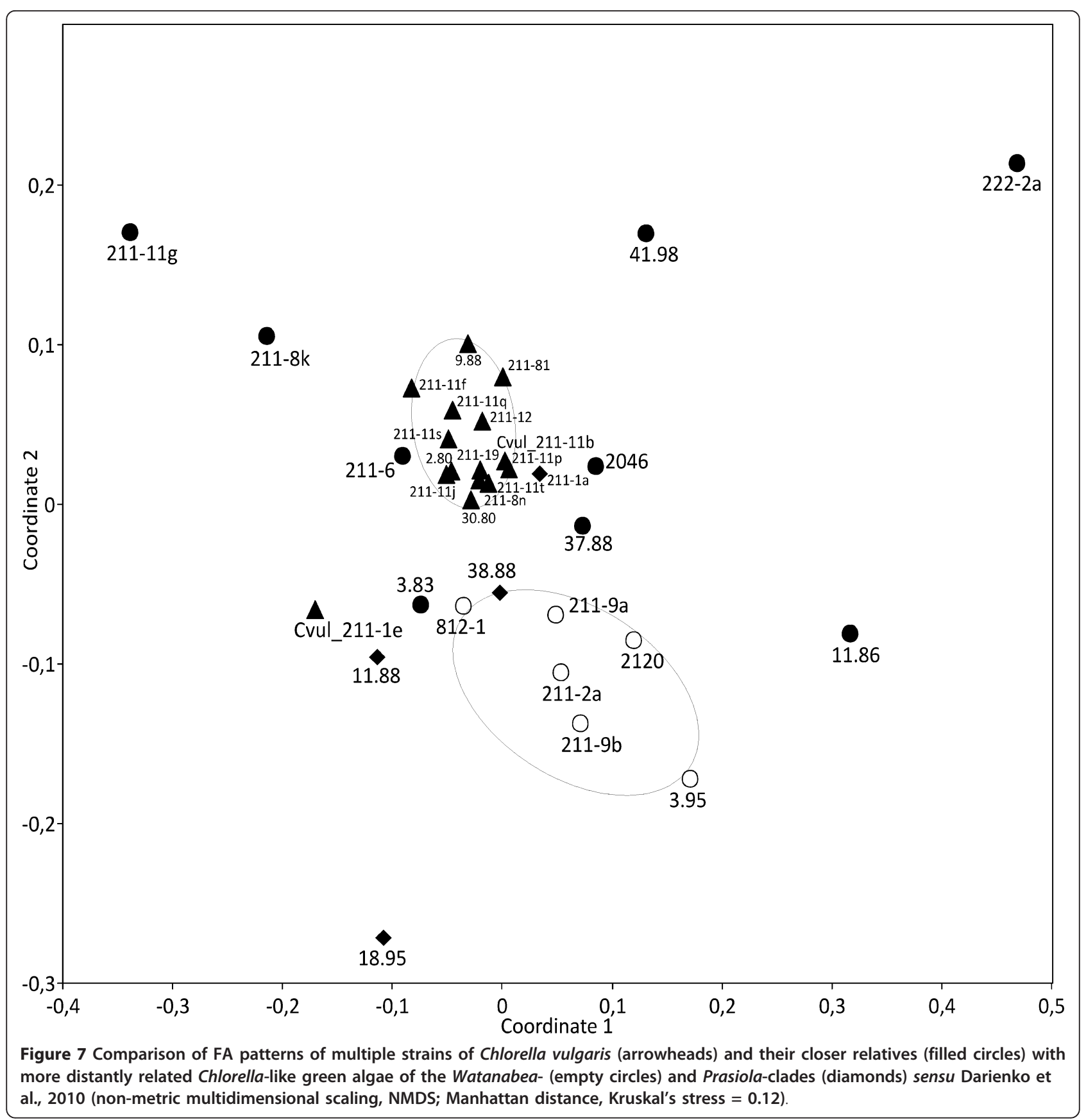

DMOX derivatives to allow identification by MS [37]. FAMEs were prepared as described, but the hexane phases were transferred into a $1.5 \mathrm{ml}$ glass tube. Samples were dried under streaming nitrogen and $200 \mu \mathrm{l} \mathrm{2-}$ alkenyl-4, 4-dimethyloxazoline (Sigma, München) were added. After incubation at $180^{\circ} \mathrm{C}$ over night in a heating block, the samples were cooled to RT and transferred with $2 \mathrm{ml}$ dichloromethane into a $12 \mathrm{ml}$ glass tube and reextracted with $5 \mathrm{ml}$ hexane and $2 \mathrm{ml}$ water. The hexane phase was dried under streaming nitrogen and redissolved with $50 \mu \mathrm{l}$ chloroform. The DMOX derivatives were separated on a $20 \mathrm{~cm} \times 20 \mathrm{~cm}$ silica gel 60 TLC plate (Merck) with petrol ether/diethyl ether 2:1 $(\mathrm{v} / \mathrm{v})$ as a developing solvent. The plate was sprayed with $0.2 \% 8$-anilino-1-naphthalene-sulfonic acid to visualize the DMOX derivatives under UV-light. The blue/yellow band of the DMOX derivatives was scraped out and the derivatives extracted by consecutive addition of $0.4 \mathrm{ml}$ water, $2 \mathrm{ml}$ methanol, $2 \mathrm{ml}$ chloroform and 2 $\mathrm{ml}$ saturated $\mathrm{NaCl}$ solution. Between each step the 
sample was vigorously mixed and finally centrifuged for $5 \mathrm{~min}$ at $3220 \times g$ to separate phases. The lower phase was transferred into a new glass tube and dried under nitrogen stream, redissolved in $10 \mu \mathrm{l}$ acetonitrile and analysed with GC/MS.

\section{Identification of FAMEs by GC with flame ionisation detection (FID)}

The prepared FAMEs were analysed by GC/FID using a capillary DB-23 column $(30 \mathrm{~m} \times 0.25 \mathrm{~mm}, 0.25 \mu \mathrm{m}$ coating thickness, J\&W, Scientific, Agilent, Waldbronn) according to Hornung et al. (2002). Helium was used as carrier gas with a flow of $0.1 \mathrm{ml} / \mathrm{min}$. The temperature gradient was $150^{\circ} \mathrm{C}$ for $1 \mathrm{~min}, 150-200^{\circ} \mathrm{C}$ at $4 \mathrm{~K} / \mathrm{min}, 200-$ $250^{\circ} \mathrm{C}$ at $5 \mathrm{~K} / \mathrm{min}$ and $250^{\circ} \mathrm{C}$ for $6 \mathrm{~min}$. Tripentadecanoate was added to each sample for quantification and the FAMEs were identified according to the retention time of the corresponding peaks in the standard "F.A.M.E. Mix C4-C24" (Sigma, München), which was injected before every $50^{\text {th }}$ run. The injection volume depended on the concentration of FAMEs within in the sample.

\section{Identification of FAMEs by GC/MS}

FID signals which were not identified by their retention times on GC and either represented FAMEs or other unpolar substances were further analysed by their mass spectra using a 6890 Gas Chromatograph/5973 Mass Selective Detector system (Agilent, Waldbronn). The $\mathrm{GC} / \mathrm{MS}$ conditions were the same as for $\mathrm{GC}$-analysis. The electron energy was $70 \mathrm{eV}$, the ion source temperature $230^{\circ} \mathrm{C}$, and the temperature for the transfer line added up to $260^{\circ} \mathrm{C}$. The identification of unknown substances was done by comparison of the obtained mass spectra with the mass spectra library NIST98 and the "Lipid Library" of the Scottish Crop Science research Institute http://www.lipidlibrary.co.uk/index.html.

\section{Analysis of FAMEs}

All chromatograms of the microalgal samples were analysed by using the ChemStation software version 9.03 (Agilent, Waldbronn). All peaks spanning a peak area of more than 50 units were integrated. The amount of each FAME was calculated using a defined amount $(1 \mu \mathrm{g})$ of the internal standard tripentadecanoate and the dry weight (DW) of each sample: area of peak $\times 1 \mu \mathrm{g} /$ area of tripentadecanoate $/ \mathrm{mg}$ d.w $=\mu \mathrm{g}$ FAME $/ \mathrm{mg}$ DW

\section{Statistical analyses of FA distribution patterns}

For each detected fatty acid (FA) its percentage of the total FA content of a strain was used as variable. For the investigation of the general structure of the data sets, common indirect ordination techniques were used, i.e. Principal Components Analysis (PCA), Correspondence and Detrended Correspondence Analysis, and Non-
Metric Multidimensional Scaling (NMDS). The significance of the differences among a priori predefined algal groups were tested using non-parametric multidimensional significance tests (Non-Parametric Multivariate Analysis of Variance, Analysis of Similarity) and visualised as ordinations from multigroup discriminant analysis (Canonical Variates Analysis). The percentages of dissimilarity between group pairs were investigated conducting SIMPER analysis. To link the significant differences among algal groups with particular variables/fatty acids possibly contributing to the observed difference, correlation analyses were conducted (Spearman's rank correlation coefficient, $\rho /$ rho), permutation significance tests). All statistical analyses and graphical visualisations have been conducted in PAST version 2.07 software package. Final graphical attributes required for publication were adapted in vector graphics editor Inkscape version 4.7 and CorelDraw X3 Graphic suite.

\section{Additional material}

Additional file 1: FAME database established of all SAG microalgal strains screened. The database contains information about clade, phylum, class, genus and species identification ( $1^{\text {st }}$ to $5^{\text {th }}$ column) as well as SAG strain number ( $6^{\text {th }}$ column) and the amount of the different substances given as relative proportion (following columns).

Additional file 2: Reduced FAME database for statistical analyses The database contains information about clade, phylum, class, genus and species identification ( $1^{\text {st }}$ to $5^{\text {th }}$ column) as well as SAG strain number $\left(6^{\text {th }}\right.$ column $)$ and the amount of the different substances given as relative proportion (following columns).

\section{List of abbreviations}

ALA: a-linolenic acid; ARA: Arachidonic acid; CVA: canonical variance analysis; DHA: docosahexaenoic acid; DMOX: 4, 4-dimethyloxaline; EPA:

Eicosapentaenoic acid; FA: fatty acid; FAME: fatty acid methyl ester; GC: gas chromatography; GLA: $\gamma$-Linolenic acid; MS: mass spectrometry; NMDS: nonmetric multidimensional scaling; PA: palmitic acid; PUFAs: polyunsaturated fatty acids; SAG: culture collection of microalgae in Göttingen; SDA: stearidonic acid.

\section{Acknowledgements and Funding}

The authors are grateful to Dr. Fredi Brühlmann (Geneva) and Dr. Cornelia Göbel (Göttingen) for their continuous support with analytical methods and interpretation of data, Dr. Maike Lorenz (Göttingen) for continuous support with microalgal handling, database work and interpretation of data, Prof. Dr. Rüdiger Hardeland (Göttingen) for providing with dinophyte strains. This work was supported by Firmenich SA, Geneva.

\section{Author details}

${ }^{1}$ Georg-August-University, Albrecht-von-Haller-Institute for Plant Sciences, Department of Plant Biochemistry, Göttingen, Germany. ${ }^{2}$ Cyano-Biofuels GmbH, Magnussstrasse 11, 12489 Berlin, Germany. ${ }^{3}$ Georg-August-University, Albrecht-von-Haller-Institute for Plant Sciences, Department of Experimental Phycology and Culture Collection of Algae in Göttingen (EPSAG), Göttingen, Germany.

\section{Authors' contributions}

IL carried out the fatty acid analysis of all algal strains and drafted the manuscript. LH performed the statistical analysis. IF and TF conceived of the study, and participated in its design and coordination and helped to draft the manuscript. All authors read and approved the final manuscript. 
Received: 6 May 2011 Accepted: 6 September 2011

Published: 6 September 2011

\section{References}

1. Bergé J-P, Barnathan G: Fatty acids from lipids of marine organisms: Molecular biodiversity, roles as biomarkers, biologically active compounds, and economical aspects. Adv Biochem Eng/Biotechnol 2005, 96:49-125

2. Dunstan GA, Volkman JK, Barret SM, Leroi J-M, Jeffrey SW: Essential polyunsaturated fatty acids from 14 species of diatom (Bacillariophyceae). Phytochemistry 1994, 35:155-161.

3. Tonon T, Harvey D, Larson TR, Graham IA: Long chain polyunsaturated fatty acid production and partitioning to triacylglycerols in four microalgae. Phytochemistry 2002, 61:15-24.

4. Viso A-C, Marty J-C: Fatty acids from 28 marine microalgae. Phytochemistry 1993, 34:1521-1533.

5. Harwood JL, Guschina IA: The versatility of algae and their lipid metabolism. Biochimie 2009, 91:679-684.

6. Watson SB: Cyanobacterial and eukaryotic algal odour compounds: signals or by-products? A review of their biological activity. Phycologia 2003, 42:332-350.

7. Mongrand S, Badoc A, Patouille B, Lacomblez C, Chavent M, Bessoule J-J: Chemotaxonomy of the Rubiaceae family based on leaf fatty acid composition. Phytochemistry 2005, 66:549-559.

8. Spitzer V: Screening analysis of unknown seed oils. Fett/Lipid 1999, 101:2-19.

9. Rossi S, Sabates A, Latasa M, Reyes E: Lipid biomarkers and trophic linkages between phytoplankton, zooplankton and anchovy (Engraulis encrasicolus) larvae in the NW Mediterranean. J Plankton Res 2006, 28:551-562.

10. Schweder T, Lindequist $U$, Lalk M: Screening for new metabolites from marine microorganisms. In Marine Biotechnology I. Volume 96. Springer Berlin/Heidelberg; 2005:1-48, Advances in Biochemical Engineering/ Biotechnology.

11. Volkman JK, Barrett SM, Blackburn SI, Mansour MP, Sikes EL, Gelin F: Microalgal biomarkers: A review of recent research developments. Org Geochem 1998, 29:1163-1179.

12. Temina M, Rezankova H, Rezanka T, Dembitsky VM: Diversity of the fatty acids of the Nostoc species and their statistical analysis. Microbiol Res 2007, 162:308-321

13. Leblond JD, Dahmen JL, Seipelt RL, Elrod-Erickson MJ, Kincaid R, Howard JC, Evens TJ, Chapman PJ: Lipid composition of chlorarachniophytes (Chlorarachniophyceae) from the genera Bigelowiella, Gymnochlora, and Lotharella. J Phycol 2005, 41:311-321.

14. Dunstan GA, Brown MR, Volkman JK: Cryptophyceae and Rhodophyceae; chemotaxonomy, phylogeny, and application. Phytochemistry 2005, 66:2557-2570.

15. Shiran D, Khozin I, Heimer YM, Cohen Z: Biosynthesis of eicosapentaenoic acid in the microalga Porphyridium cruentum.1. The use of externally supplied fatty acids. Lipids 1996, 31:1277-1282.

16. Cohen Z: The production potential of eicosapentaenoic and arachidonic acids by the red alga Porphyridium cruentum. J Am Oil Chem Soc 1990 67:916-920.

17. Zook D, Schenk HEA: Lipids in cyanophora paradoxa. III. Lipids in cell compartments. Endocyt C Res 1986, 3:203-211.

18. Cavalier-Smith T: The origin of eukaryotic and archaebacterial cells. Ann N Y Acad Sci 1987, 503:17-54

19. Neustupa J, Elias M, Skaloud P, Nemcova Y, Sejnohova L: Xylochloris irregularis gen. et sp. nov. (Trebouxiophyceae, Chlorophyta), a novel subaerial coccoid green alga. Phycologia 2011, 50:57-66.

20. Bigogno C, Khozin-Goldberg I, Boussiba S, Vonshak A, Cohen Z: Lipid and fatty acid composition of the green oleaginous alga Parietochloris incisa, the richest plant source of arachidonic acid. Phytochemistry 2002, 60:497-503.

21. Caudales R, Wells JM: Differentiation of free-living Anabaena and Nostoc cyanobacteria on the basis of fatty-acid composition. Int I Syst Bacteriol 1992, 42:246-251.

22. Krüger $\mathrm{G}$, De Wet $\mathrm{H}$, Kock J, Pieterse A: Fatty acid composition as a taxonomic characteristic for Microcystis and other coccoid cyanobacteria (blue-green alga) isolates. Hydrobiologia 1995, 308:145-151.
23. Dembitsky VM, Srebnik M: Variability of hydrocarbon and fatty acid components in cultures of the filamentous cyanobacterium Scytonema $\mathrm{sp}$. isolated from microbial community "black cover" of limestone walls in Jerusalem. Biochemistry (Mosc) 2002, 67:1276-1282.

24. Kenyon CN: Fatty acid composition of unicellular strains of blue-green algae. J Bacteriol 1972, 109:827-834

25. Kenyon CN, Stanier RY: Possible evolutionary significance of polyunsaturated fatty acids in blue-green algae. Nature 1970, 227:1164-1166.

26. Keeling PJ: Diversity and evolutionary history of plastids and their hosts. Am J Bot 2004, 91:1481-1493.

27. Gould SB, Waller RF, McFadden Gl: Plastid evolution. Annu Rev Plant Biol 2008, 59:491-517.

28. Friedl T: The evolution of the green algae. Plant Syst Evol 1997, 11:87-101.

29. Lewis LA, MCCourt RM: Green algae and the origin of land plants. Am J Bot 2004, 91:1535-1556.

30. Pröschold T, Marin B, Schlosser UG, Melkonian M: Molecular phylogeny and taxonomic revision of Chlamydomonas (Chlorophyta). I. Emendation of Chlamydomonas Ehrenberg and Chloromonas Gobi, and description of Oogamochlamys gen. nov. and Lobochlamys gen. nov. Protist 2001, 152:265-300.

31. Elias M, Nemcova Y, Skaloud P, Neustupa J, Kaufnerova V, Sejnohova L: Hylodesmus singaporensis gen. et sp. nov., a new autosporic subaerial green alga (Scenedesmaceae, Chlorophyta) from Singapore. Int J Syst Evol Microbiol 2010, 60:1224-1235.

32. Hegewald E, Wolf M: Phylogenetic relationships of Scenedesmus and Acutodesmus (Chlorophyta, Chlorophyceae) as inferred from 18S rDNA and ITS-2 sequence comparisons. Plant Syst Evol 2003, 241:185-191.

33. Müller J, Friedl T, Hepperle D, Lorenz M, Day JG: Distinction between multiple isolates of Chlorella vulgaris (Chlorophyta, Trebouxiophyceae) and testing for conspecificity using Amplified Fragment Length Polymorphism and its rDNA sequences. J Phycol 2005, 41:1236-1247.

34. Krienitz L, Hegewald EH, Hepperle D, Huss VAR, Rohrs T, Wolf M: Phylogenetic relationship of Chlorella and Parachlorella gen. nov (Chlorophyta, Trebouxiophyceae). Phycologia 2004, 43:529-542.

35. Pröschold T, Darienko T, Gustavs L, Mudimu O, Menendez CR, Schumann R, Karsten U, Friedl T: Chloroidium, a common terrestrial coccoid green alga previously assigned to Chlorella (Trebouxiophyceae, Chlorophyta). Eur J Phycol 2010, 45:79-95.

36. Hornung E, Pernstich $C$, Feussner I: Formation of conjugated $\Delta^{11} \Delta^{13}$ double bonds by $\Delta^{2}$-linoleic acid $(1,4)$-acyl-lipid-desaturase in pomegranate seeds. Eur J Biochem 2002, 269:4852-4859.

37. Fay L, Richli U: Location of double bonds in polyunsaturated fatty acids by gas chromatography-mass spectrometry after 4, 4-dimethyloxazoline derivatization. J Chromatogr A 1991, 541:89-98.

doi:10.1186/1471-2229-11-124

Cite this article as: Lang et al:. Fatty acid profiles and their distribution patterns in microalgae: a comprehensive analysis of more than 2000 strains from the SAG culture collection. BMC Plant Biology 2011 11:124.

\section{Submit your next manuscript to BioMed Central and take full advantage of:}

- Convenient online submission

- Thorough peer review

- No space constraints or color figure charges

- Immediate publication on acceptance

- Inclusion in PubMed, CAS, Scopus and Google Scholar

- Research which is freely available for redistribution 